Estanislau ROCA \& Miquel MARTí

Barcelona has been since the beginning of the eighties an exceptional laboratory in order to study the public space renewal, through both redesign processes and strategic urban projects. Meanwhile, the Architectural School of Barcelona has tied its teaching and research to the urban transformation of the city, which has produced a rich reflection on urban projects, through courses and publications. A result of this theoretical framework were some $\mathrm{PhD}$ dealing with public space, some of them concerning the recovering of more traditional public space in the compact city, some others studying new forms of open space in the metropolitan context. Following these first doctoral researches, a specific line on public space research was created. A comparative study on public space policies in European cities has been carried on and several $\mathrm{PhD}$ on current issues about public space have been started. Some of the studied subjects are: collective spaces in leisure artefacts, infrastructural nodes in metropolitan networks, large open spaces in river valleys, hybrid spaces in Asian cities, civic meaning of contemporary social spaces...

Key words: public space, collective space, infrastructural space, metropolitan open space, civic meanings 


\section{SOBRE ESPACIO PÚBLICO Y SU PROYECTO URBANO}

Estanislau ROCA y Miquel MARTí

Barcelona ha sido desde principios de los años ochenta un excepcional laboratorio para el estudio de la renovación del espacio público, especialmente a través de los procesos de reurbanización y de proyectos urbanos estratégicos. Al mismo tiempo, desde la Escuela Técnica Superior de Arquitectura de Barcelona se ha vinculado estrechamente la docencia y la investigación con la transformación urbanística de la ciudad, dando lugar a una rica reflexión, tanto a través de asignaturas, como de publicaciones sobre el proyecto urbano. Fruto de este marco de reflexión fueron unas primeras tesis doctorales dedicadas al tema del espacio público, tanto a la recuperación de los espacios más tradicionales en las ciudades compactas, cómo sobre el potencial de nuevas formas de espacios libres en el contexto metropolitano. A partir de éstas, se impulsó una línea específica de investigación sobre el espacio público, que ha propiciado la realización de una investigación sobre políticas de espacio público en ciudades europeas y el arranque de otras investigaciones sobre los espacios públicos: los espacio colectivos en artefactos del ocio, los nodos infraestructurales en redes metropolitanas, los grandes espacios libres de corredores fluviales, los espacios híbridos en ciudades asiáticas o los significados cívicos de los espacios de encuentro.

Palabras clave: espacio público, espacio colectivo, espacio infraestructural, espacio libre metropolitano, sentido cívico 


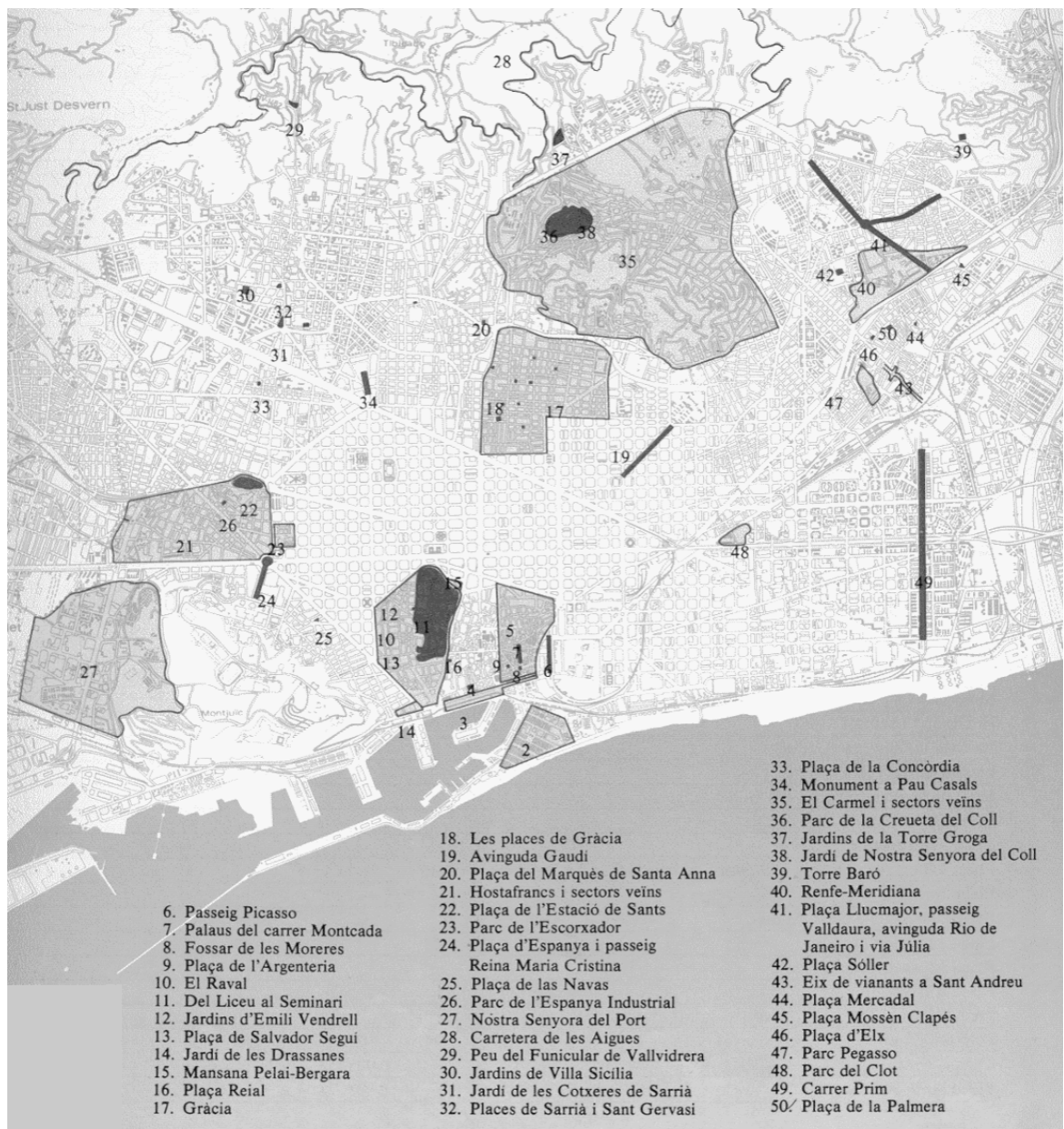

fig. 1 Planes y proyectos estratégicos para Barcelona en los años ochenta 


\section{BARCELONA COMO LABORATORIO: Una experiencia continuada en el proyecto urbano y la transformación del espacio público}

Con la recuperación de los Ayuntamientos democráticos, y bajo la batuta de Oriol Bohigas desde el Consistorio, y la inspiración conceptual del Departamento de Urbanismo y Ordenación del Territorio de la UPC, con Manuel de Solà-Morales al frente, Barcelona inicia, a comienzos de los años ochenta del siglo pasado, una profunda transformación urbanística, que continúa abierta y candente tres décadas después.

La idea de espacio público ha jugado un rol central en el reciente desarrollo urbanístico de la ciudad, entendiendo por espacio público aquel lugar intensamente vivido, que propicia ricas interacciones psicológicas y sociales en el acto de exponerse en público (y que, por lo tanto, puede darse no sólo en el espacio libre de propiedad pública, sino también en espacios cerrados y/o de propiedad privada). Desde la búsqueda, en los primeros ochenta, de monumentalidad, de espacios públicos claramente identificables y memorables para la ciudadanía, tanto en el centro, como en la periferia, hasta los espacios públicos dotados de nuevas tecnologías que intentan dialogar, hoy en día, con el patrimonio industrial en el distrito 22@, el espacio público no ha parado de estar en el corazón de la reforma urbana de Barcelona, hasta convertirla en una de las ciudades con espacio público de mejor calidad, y eso, en toda la extensión de la ciudad.

Esta mejora del espacio público se ha alcanzado mediante una doble política de reurbanización y de proyectos urbanos estratégicos. Desde las creaciones iniciales de nuevos espacios públicos, aprovechando a menudo la oportunidad de solares adquiridos por el Ayuntamiento desde la década de los setenta (plazas como la Mercè en el corazón de Ciutat Vella, Sóller en Nou Barris o parques como la Espanya Industrial, l'Escorxador o la Pegaso), pasando por la reforma de las principales avenidas de la ciudad (de la Avenida Meridiana a los bulevares de Nou Barris o la Ronda del Mig), hasta la mejora extensiva del entramado de calles en muchos barrios de la ciudad, la política de reurbanización ha sido un elemento crucial en la mejora del espacio público.

Crucial fue también el giro copernicano en la manera de proyectar y entender el espacio público de la ciudad de Barcelona que imprimió Oriol Bohigas. Aquel otro urbanismo de reforma, el paso del Plan al Proyecto, la renovación de barrios y la concepción de otra urbanidad, caracterizaron un episodio importante de la historia del urbanismo de nuestra ciudad. Barcelona tenía que ser regenerada a iniciativa de la propia Administración, directamente 
o haciendo otra vez y sectorialmente rentable la intervención privada. Paulatinamente, el proyecto urbano se convierte en motor de la renovación urbana de la ciudad y de su espacio público.

Bohigas apostaba por proyectos muy diferentes a la simple concreción de la vialidad, y aducía que hacían falta proyectos integrales que tenían que resolver, cada uno de ellos, problemas concretos que hiciesen arrancar una cierta dinámica propia dentro de las posibilidades existentes. Era el retorno de la idea de la calle, la plaza y los jardines urbanos, conformados por la arquitectura, la arquitectura de la ciudad, que tenía como incitador, con aportaciones propias muy considerables, tanto propositivas como pedagógicas, nuestro Departamento de Urbanismo y Ordenación del Territorio. Junto con Manuel de Solà-Morales, cabe destacar sobretodo a Joan Busquets, quien pronto se hizo cargo de la dirección urbanística de la ciudad y fue el responsable de los planes de transformación más relevantes, entre los que destacan el Plan de Vías y el de Áreas de nueva centralidad.

En este nuevo contexto, los arquitectos tuvieron mucho más protagonismo en las obras públicas municipales y Barcelona fue mejorando poco a poco hasta alcanzar una notable calidad en su diseño urbano.

Durante las tres últimas décadas la ciudad se ha transformado cualitativamente y, además, ha doblado la superficie de zonas verdes. Se ha convertido en un verdadero laboratorio de proyectos urbanos, que culminó, en una primera fase, con la ciudad reformada para acoger los Juegos Olímpicos de 1992, seguida de una segunda renovación hasta 1995, caracterizada por la continuación de los proyectos anteriores, al mismo tiempo que se pusieron en marcha grandes proyectos, entre ellos la transformación de la Maquinista y la Sagrera, con la preparación de la reforma urbana para la estación de tren de alta velocidad. También ha experimentado una rotunda transformación el tramo final de la Diagonal y su llegada al mar mediante la operación que permitió la celebración del Fórum de las Culturas de 2004. Éste ha sido sin duda el episodio de reforma urbana menos afortunado, fruto de haber confiado excesivamente en la iniciativa privada y por ello con un menor control por parte del Ayuntamiento. Y ha seguido con la transformación del Distrito 22@ del Poble Nou, un buen exponente de lo que será la revolución industrial del siglo XXI, la tecnología. En él nos encontramos con un "collage" de brillantes actuaciones, combinando industrias de nuevas tecnologías y residencias, y el espacio público que dialoga con ellas y con el patrimonio histórico industrial.

Desde el principio, se ha trabajado con una actitud estratégica o de acupuntura urbana, buscando con cada proyecto un valor añadido más allá de su estricto ámbito. Este carácter estratégico de las actuaciones de reforma destaca en los barrios más desatendidos históricamente. Mientras que, en paralelo, se contrataba a los mejores escultores contemporáneos para que sus obras presidieran nuevos espacios públicos. De esta manera, implícitamente se ponía en el mapa cultural y turístico nuevos fragmentos de la ciudad. Y, ahora, no es estraño encontrarnos con obras de Ellswort Kelly, Bryan Hunt, Eduardo Chillida, Richard Serra, Antoni Tàpies, Anthony Caro, Rebecca Horn o de Claes Oldenburg, entre otros. 
Toda esta casuística nos ha legado un escenario en el que legibilidad, coexistencia de usos diversos, comprensión de la estructura urbana, signos de identidad colectiva y complejidad, condiciones del carácter cívico del espacio urbano, se encuentran reforzados en un proceso de transformación del espacio público presidido por lógicas urbanas. Es justamente la imagen de este espacio público plenamente urbano el que añade a la experiencia de la alteridad y de la propia individualidad que propicia toda interacción social, la experiencia de la colectividad. ${ }^{1}$

Como resultado, Barcelona hoy puede presumir de una gran cantidad de excelentes lecciones en la conceptualización del espacio público y proyectos urbanos merecedores de los reconocimientos más destacados, como los premios del Royal Institute of British Architects y de la Universidad de Harvard, entre otros, convirtiéndose en un referente mundial del urbanismo, la arquitectura y el diseño urbano. Barcelona es, en este sentido, un laboratorio privilegiado para el estudio del proyecto urbano y las transformaciones del espacio público.

\section{LA DOCENCIA EN LA ETSAB: EL FEEDBACK PERMANENTE CON LA REALIDAD. De las reflexiones de Manuel de Solà Morales sobre el proyecto urbano a múltiples asignaturas}

Con la transformación urbanística de Barcelona como telón de fondo, en la Escuela Técnica Superior de Arquitectura, ya desde la elaboración del Plan de Estudios de 1979, se ha defendido la fusión entre urbanismo y arquitectura como campos intelectual y profesionalmente inseparables en nuestro contexto ${ }^{2}$. Una tal unión viene a confirmar la apuesta por aquel "urbanismo para arquitectos" que distinguía la orientación de nuestra Escuela del modelo anglosajón escalonado, de los ensayos italianos de una nueva carrera urbanística y de la trilogía urbanismo-arquitectura-diseño de raíces bauhasianas, entonces imperantes en el marco cultural europeo y americano. Esto viene asimismo a validar las conclusiones que se deducen tras un profundo y detallado análisis de la enseñanza del urbanismo elaborado por el Departamento de Urbanismo y Ordenación del Territorio a partir de 1970 como consecuencia de un premio a la investigación de la Fundación Juan March³

Formación universitaria única e idéntica calificación para el ejercicio profesional son condicionantes básicos de la organización de una enseñanza que acentúa igualmente la reflexión crítica y la capacitación proyectual. El tradicionalmente defendido "urbanismo para arquitectos", como apuesta por una línea disciplinar, exigió desde el principio una definición pedagógica que enfatizara un campo de reflexión, unos métodos de análisis y asimismo unos instrumentos de intervención propios.

\footnotetext{
1 Miquel Martí en "A la recerca de la civitas contemporània. Cap a una cultura urbana de l'espai públic: l'experiència de Barcelona (1979-2003)" Tesis doctoral inédita. 2004.

2 Cuestión que vuelve a plantease con idéntica respuesta en el Congreso de Arquitectos de Granada (1981) y en las jornadas sobre enseñanza de la Arquitectura en Lloret de Mar (1986); y que tras los imprescindibles ajustes que ha implicado la incorporación española a la CEE (homologación de títulos) ha vuelto sin duda a surgir en la discusión de los nuevos planes de estudios universitarios.

3 Manuel de Solá Morales Rubió y Laboratorio de Urbanismo: La enseñanza del Urbanismo (3 volúmenes): I. Situación intenacional, II. Situación española, III. Resumen y Conclusiones. ETSAB. Barcelona 1974.
} 
Pero con el tiempo el desarrollo de las enseñanzas de urbanismo desde el segundo curso hasta el Proyecto Fin de Carrera se ha abierto una nueva vía a aquellos estudiantes interesados: la posibilidad de una cierta profundización urbanística que se orienta hacia una mayor capacitación profesional en la intervención en la ciudad.

Resumidamente, durante las últimas décadas la enseñanza del urbanismo en la ETSAB ha estado enfocada a la formación del estudiante para la comprensión de la ciudad, de su forma -en tanto que reflejo de valores sociales y culturales-, de su proyecto -como resultado de intenciones, técnica e instrumentos-, de las partes y elementos componentes, y de las relaciones entre ellas. Al mismo tiempo se ha dirigido a la capacitación del futuro arquitecto en su trabajo de proyectista, que comprende la disposición de las calles, agrupación de las construcciones, diseño del suelo y de los espacios construidos, configuración de paisajes y áreas urbanas.

En los últimos planes de Estudios se han ajustado estos objetivos en seis cuatrimestres, en los cuales van variando los métodos docentes y sistemas de evaluación. La implantación de cada Plan de Estudios ha supuesto una oportunidad de actualización de contenidos y la renovación de la orientación pedagógica.

El estudio de la ciudad y sus elementos de las formas de crecimiento e ideas de intervención; del proyecto de las calles y de la edificación; del proyecto de la estructura urbana y del proyecto urbano ha sido el contenido principal de los cursos de grado en la ETSAB, y desde siempre encontramos el liderazgo del catedrático Manuel de Solà Morales, director del Laboratorio de Urbanismo de Barcelona (LUB) desde 1968.

Su valiosa aportación en la docencia de los distintos cursos ha permitido ir construyendo un background que hemos podido completar año tras año y adaptarlo a las necesidades de la sociedad.

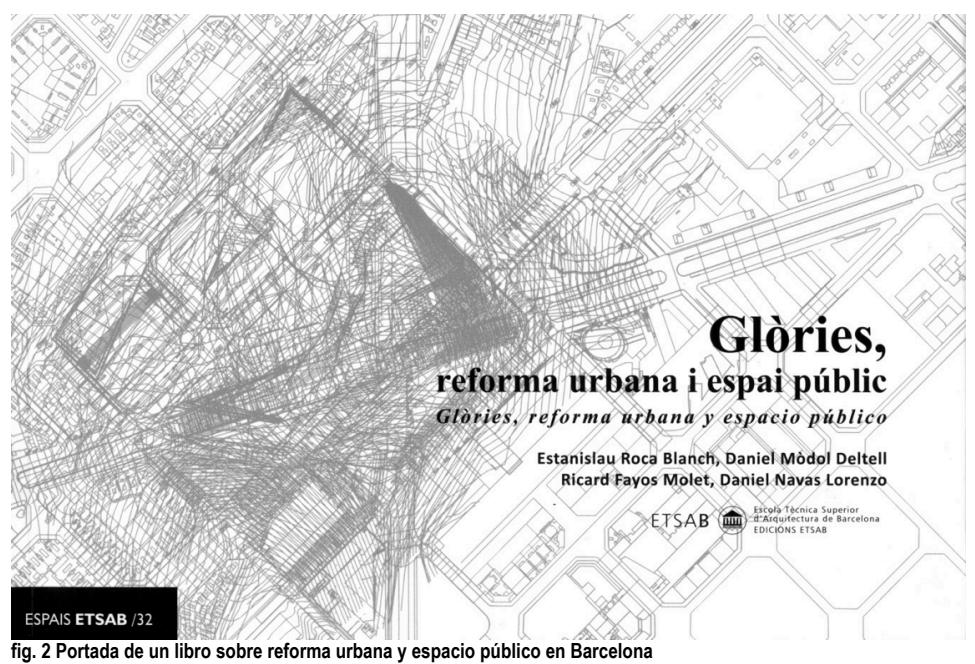

fig. 2 Portada de un libro sobre reforma urbana y espacio público en Barcelona 
Las lecciones de Manuel de Solà Morales, sus escritos e ideas han fomentado un campo de investigación muy rico del que se han derivado excelentes tesis doctorales sobre el proyecto de ciudad.

Cabe resaltar la conferencia inaugural de nuestro Máster de Proyectación Urbanística del 6/03/1995 y la conferencia inaugural del curso académico de la ETSAB de 2009/20104. Tanto en una como en la otra, nos seducía con sutiles líneas e ideas sobre la responsable intervención del urbanista en la ciudad y los conceptos de urbanidad en el proyecto urbano.

Todo ello ha derivado en los enfoques sobre el proyecto urbano del espacio público y/o colectivo en asignaturas de grado y de postgrado. Como también encontramos numerosos escritos y publicaciones fruto de la producción docente de estos años. ${ }^{5}$

De todas estas publicaciones cabe destacar el compendio de textos seleccionados por Manuel de Solà Morales en "De cosas urbanas", el primero corresponde a la conferencia impartida en la UQÀM (Université du Québec à Montreal), de septiembre de 2005 y los otros habían aparecido con anterioridad en distintas publicaciones ${ }^{6}$ :

El Departamento de Urbanismo y Ordenación del Territorio ha sido clave en la gran transformación de la ciudad de Barcelona desde los años 80 del siglo pasado y la ciudad se ha convertido en un verdadero laboratorio de proyectos urbanos, fruto de la continua y mutua transferencia de conocimiento entre el mundo docente y el profesional.

\footnotetext{
${ }_{4}^{4}$ Publicada en Visions 8. La urbanitat de l'arquitectura. Conferència inaugural del curs 2009-2010

5 - AAVV. El Projecte urbà: una experiència docent. Barcelona: Edicions UPC, 1999.

- AAVV. Ciutat funcional i morfologia urbana. Barcelona: Ed. UPC, 1994.

- AAVV. Ceuta: La estrategia de un lugar. ETSAB, ETSALS i ESARQ. Instituto d'estudios ceutíes, 2010.

- AAVV. Publicada en Visions 8. La urbanitat de l'arquitectura. Conferència inaugural del curs 2009-2010. Barcelona: Ed. ETSAB, 2010.

- Martí, Miquel. A la recerca de la civitas contemporània. Cap a una cultura urbana de l'espai públic: l'experiència de Barcelona. DUOT (Tesis Doctoral)

- Roca, Estanislau et alt. Campus de la Diagonal. Un projecte urbà: praxi docent i professional. Barcelolna: Edicions UB, 2009.

- Roca, Estanislau et alt. Diagonal, un procés urbà: el projecte urbà en la reforma de la ciutat. Barcelona: Edicions ETSAB, 2010.

- Roca, Estanislau et alt. Glòries, reforma urbana i espai públic. Barcelona: Edicions ETSAB, 2011.

- Roca, Estanislau; Mòdol, Dani; Franco, Manuel. EI Projecte de l'espai viari. Barcelona: Edicions UPC, 2004.

- Solà-Morales i Rubió, Manuel. Cuatro líneas, tres artículos, siete ideas: las formas de la proyectación urbanística, en: 'Proyectar la ciudad : programa de asignaturas : marzo-mayo 1995'. Barcelona : [S.n.], 1995.

- Solà-Morales i Rubió. Manuel. Espacios públicos y espacios colectivos: un nuevo reto, urbanizar lo privado. 'La Vanguardia', Suplemento 12/05/1992. Pág 4 i 5

- Solà-Morales, Manuel. De cosas urbanas. Barcelona: Gustavo Gili, 2008.

- El Proyecto Urbano I. 'UR: urbanismo revista: publicación internacional periódica del Laboratorio de Urbanismo de Barcelona', 1987, núm. 5. Disponible a: <http://upcommons.upc.edu/revistes/handle/2099/3100>

- El proyecto Urbano II. 'UR: urbanismo revista: publicación internacional periódica del Laboratorio de Urbanismo de Barcelona', 1988, núm. 6. Disponible a:<http://upcommons.upc.edu/revistes/handle/2099/3155>

6 - Para una urbanidad material. (2005)

- Contra la metrópoli universal. (2000)

- Territorios sin modelo. (1995)

- Ciudades cortadas. (1994)

- Espacios públicos / espaciosa colectivos. (1992)

- La periferia como proyecto. (1992)

- Otra tradición moderna. (1987)
} 


\section{TESIS DOCTORALES PIONERAS EN EL ESTUDIO DEL ESPACIO PÚBLICO}

Entre un proyecto de ciudad que daba mucha importancia al espacio público y una docencia del Departamento de Urbanismo de la UPC que analizaba y teorizaba esta experiencia de transformación urbana, interesándose especialmente por el proyecto urbano, surgen distintas tesis doctorales relacionadas con esta temática. Fueron el embrión de lo que sería una línea de investigación sobre espacio público con entidad propia dentro del Programa de Doctorado en Urbanismo. Podemos hablar de cuatro tesis pioneras en este campo: las de Josep $M^{a}$ Fortià, Enric Batlle, Jaume Barnada y Miquel Martí.

La tesis de Pep Fortià fue presentada en 1999 bajo la dirección de Joaquim Español y el título de "La exploración del plano horizontal". En ella, el autor estudia el periodo de transformación del espacio público en Barcelona y otras ciudades catalanas entre 1981 y 1987. Entre la recuperación de los Ayuntamientos democráticos y la nominación de Barcelona como sede olímpica, tiene lugar un intenso proceso de renovación del espacio público con características propias y singulares. Se puede hablar del nacimiento de una escuela de espacio público barcelonesa, y más adelante catalana, que tendrá incidencia sobre el futuro del espacio público en las décadas siguientes y se erigirá en referencia internacional. La tesis muestra como los proyectos de este periodo se caracterizan por un cierto ensimismamiento que contrasta con la tradición europea de un espacio escénico de la vida urbana. Los proyectos marcan claramente sus propios límites, diferenciándose y separándose del contexto urbano. Los nuevos espacios públicos se conciben como obras de arte en sí mismos y en muchos de los casos incorporan directamente elementos escultóricos. Juegan con el paisaje cómo soporte y son ricos en alusiones metafóricas y simbólicas. En este sentido, la tesis compara los espacios catalanes con algunos ejemplos de Estados Unidos, para ver hasta que punto la nueva escuela bebe de fuentes norteamericanas, muy influidas en aquel momento por la corriente paisajística del land art.

La tesis d'Enric Batlle, dirigida por Antonio Font, se presentó en 2002 con el título "El jardí de la metròpolis". Sobre la base de una revisión de las principales aportaciones históricas sobre el proyecto del espacio libre (desde los sistemas de vías-parque norteamericanos hasta los mosaicos territoriales de la ecología del paisaje, pasando por los jardines de Burle Marx), la tesis defiende el papel del estrato de los espacios libres como vertebrador potencial de la ciudad extendida por el territorio. Este estrato del espacio libre estaría formado por todos los vacíos generados por la ocupación del territorio: los drenajes del territorio, bosques y espacios agrícolas en el entorno urbano, "infraestructuras verdes" ("paisaje resultante de una correcta implantación de las infraestructuras, capaces de recuperar toda su urbanidad") y "jardines de asignación" (espacios con intervenciones proyectuales para la implantación de usos de ocio, medioambientales o servicios). El estrato libre se complementa con la matriz ecológica de R. T. Forman y le añade la idea de su articulación con los espacios públicos urbanos (portadores de valores cívicos) y de la presencia de entornos proyectados con valores añadidos (como serían las infraestructuras verdes y los jardines de asignación). 


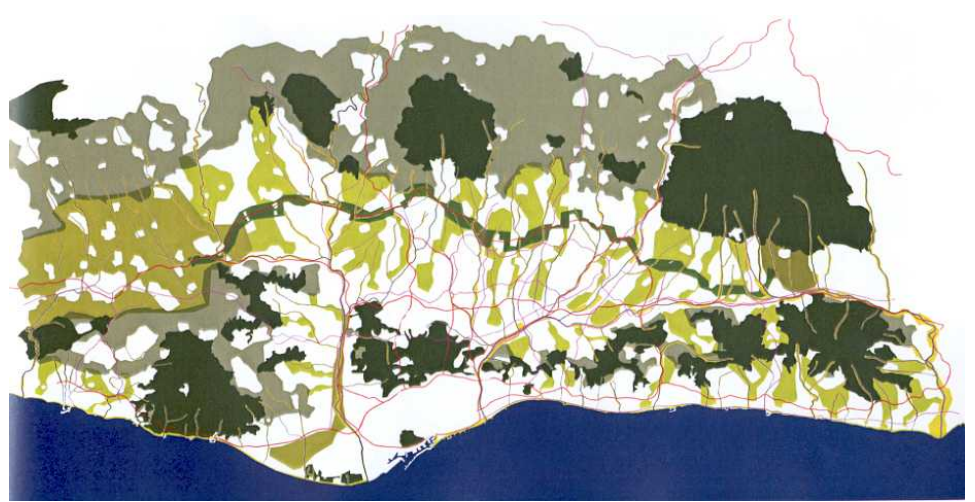

fig. 3 Espacios libres e infraestructuras metropolitanas de Barcelona en la tesis de Enric Batlle

También en 2002, fue presentada la tesis de Jaume Barnada en el Departamento de Composición Arquitectónica bajo la dirección de Carles Martí. Su título: "La ciutat com a diagrama de lloc publics". Se trata de una reflexión sobre los componentes de la estructura urbana. A partir del análisis de 12 ciudades de contextos culturales diversos (4 asiáticas, 4 europeas y 4 americanas), la tesis plantea como toda ciudad puede entenderse cómo estructurada por un diagrama de lugares públicos significativos. La identificación de estos lugares públicos y la elaboración de estos diagramas para cada ciudad, es una parte central de la investigación propuesta. Se desprende que hay dos grandes tipos de diagramas, los que articulan componentes más tradicionales cómo la calle y la plaza, y los que siguen un modelo de "campus", una ciudad más abierta y más articulada con la naturaleza.

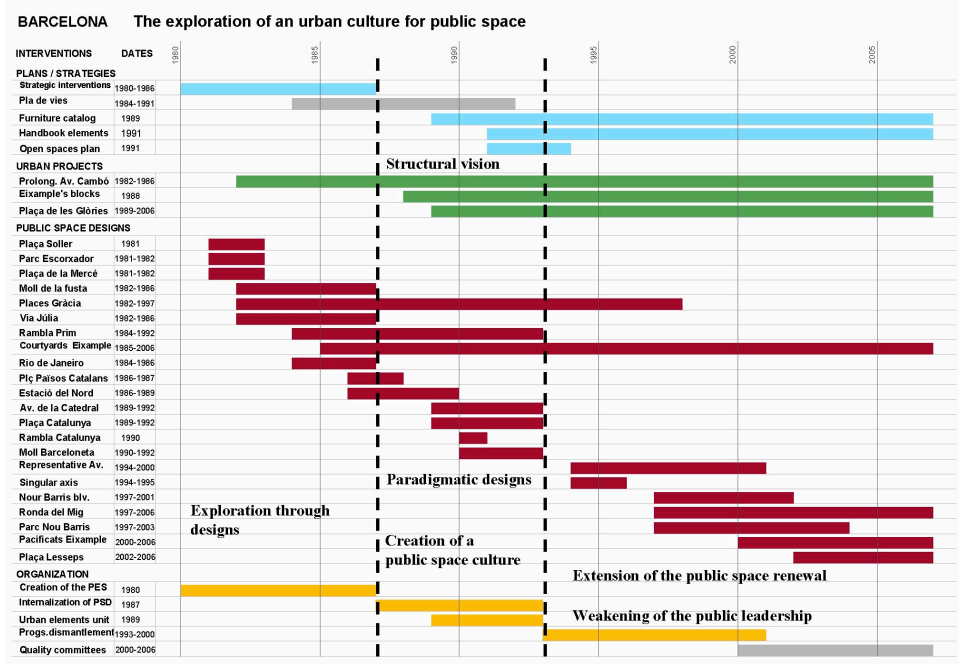

fig. 4 Cuadro síntesis de las intervenciones más significativas de renovación del espacio público en Barcelona de 1979 a 2003 


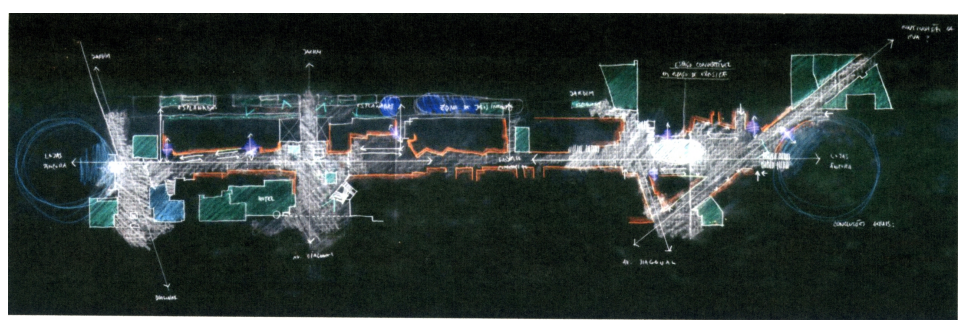

fig. 5 Estudio de l'llla Diagonal según el trabajo doctoral de Pedro Bento

La tesis de Miquel Martí, dirigida por Joaquim Sabaté, se presentó en 2004 bajo el título "A la recerca de la civitas contemporània. Cap a una cultura urbana de l'espai públic: l'experiència de Barcelona (1979-2003)". Cómo el propio título indica, se trata de un análisis de la evolución del proceso de renovación del espacio público en Barcelona desde la recuperación de los Ayuntamientos democráticos hasta el momento de presentación de la tesis, en vísperas de la celebración del Fórum 2004. El trabajo muestra cómo las exploraciones proyectuales de los primeros ochenta (ya estudiadas en la tesis de José $\mathrm{M}^{a}$ Fortià) dieron lugar a un aprendizaje y a una inflexión en el modo de proyectar el espacio público. De espacios ensimismados, se pasó a espacios que buscaban una mayor integración espacial y una mayor articulación con el entorno urbano. En la consolidación de la política de espacio público que se produjo durante el periodo de preparación de los Juegos Olímpicos (1987-1992), los nuevos espacios trabajan con los límites internos y externos y alcanzan una máxima integración, tanto interior (evitando fragmentaciones), como exterior (relacionando el proyecto con el entorno edificado).

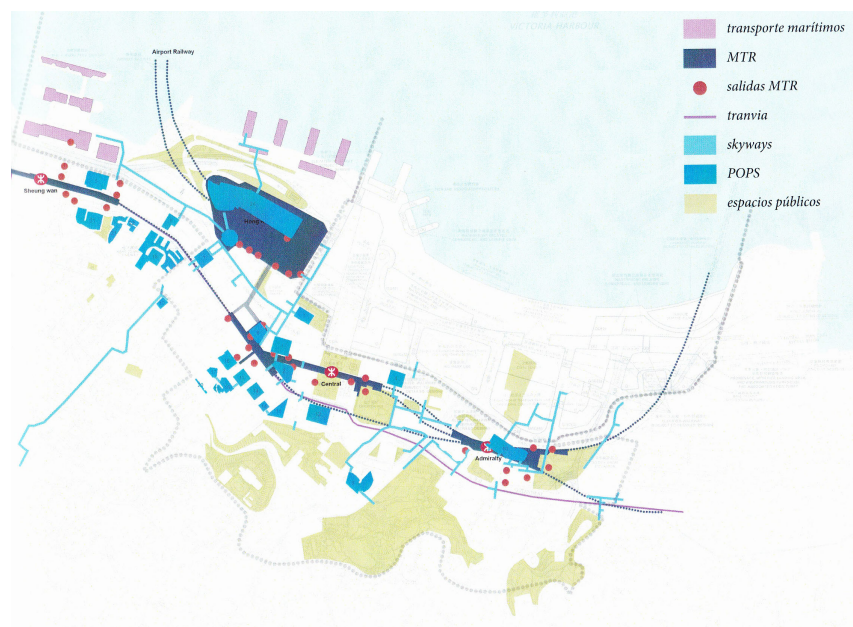

fig. 6 Espacios híbridos público-privados en Hong Kong según el trabajo doctoral de Francesco Rossini 
También se impulsará una visión más estructural de la renovación del espacio público, haciendo especial hincapié en las actuaciones de conexión entre espacios. Se pasa de intervenciones aisladas, a la reurbanización de redes de avenidas y calles, del proyecto puntual al proyecto lineal. Mediante la uniformización del lenguaje de elementos urbanos se logra también proyectar el espacio público de áreas enteras de la ciudad, creando imágenes urbanas que se van solapando a diferentes escalas (la calle, el barrio, el distrito, la ciudad). Se trata de un conjunto de cambios que acentúan la lógica urbana del proyecto del espacio público, el tener en cuenta el papel de cada espacio dentro del conjunto de la ciudad y cómo en él convergen los diferentes componentes urbanos. Es la búsqueda de una mayor urbanidad del espacio público, lo que supone reforzar su significación cívica contemporánea.

\section{PRIMERAS INVESTIGACIONES DE UNA LÍNEA SOBRE ESPACIO PÚBLICO Y PROYECTO URBANO}

Sobre la base de las tesis mencionadas, pioneras en el estudio del espacio público en la UPC, se planteó la posibilidad de estructurar una línea de investigación sobre el tema. Se concibió cómo una sublínea dentro del ámbito del Análisis y Proyecto Urbano.

Las primeras investigaciones de la nueva línea se orientaron en dos direcciones. Por un lado, la profundización del estudio de los espacios urbanos en ciudades compactas iniciado con las tesis de Josep $\mathrm{M}^{\mathrm{a}}$ Fortià, Jaume Barnada y Miquel Martí. Por otro lado, la realización de tesis doctorales sobre nuevas tipologías de espacios públicos, algunas de las cuales (cómo las "Infraestructuras verdes") jugaban un papel central en la tesis d'Enric Batlle.

En la profundización del estudio del espacio público urbano en ciudades compactas se procedió a realizar una investigación comparativa entre nueve ciudades europeas que permitía contextualizar la experiencia de Barcelona y obtener una visión panorámica sobre la situación del espacio público en Europa. Además de Barcelona, las nueve ciudades escogidas fueron Ámsterdam, La Haya y Róterdam en los Países Bajos, París y Lyon en Francia y Berlín, Roma y Sevilla. Se eligieron ciudades que habían impulsado procesos de renovación del espacio público y representativas de situaciones nacionales diferentes. La investigación, financiada por la Universidad TU Delft bajo la dirección de Miquel Martí, se realizó entre 2005 y 2007.

La evolución del caso de Barcelona había puesto de manifiesto que las renovaciones de espacio público eran procesos generales que difícilmente podían ser valorados simplemente a partir del estudio de proyectos puntuales desligados entre sí. De este modo, para cada ciudad estudiada se empezó elaborando un cuadro síntesis de las actuaciones más representativas en la transformación del espacio público. Al lado de los proyectos de reurbanización, se incluyeron explícitamente los proyectos urbanos que configuraban espacios 
públicos desde el trabajo con los entornos edificados y los planes o estrategias (desde planes para la renovación del espacio público de áreas determinadas de la ciudad como podrían ser centros históricos, hasta estrategias para extender un lenguaje de mobiliario específico en toda la ciudad). El caso de Barcelona había mostrado también la importancia de los cambios organizativos de los agentes que protagonizan la renovación del espacio público, por lo cual, para cada ciudad se identificaron también las formas de gestión de estos procesos y sus cambios. Los cuadros síntesis elaborados con todas estas intervenciones o cambios organizativos situados cronológicamente desde el momento en qué la ciudad había emprendido explícitamente la mejora de su espacio público permiten construir la visión global sobre estos procesos que se buscaba.

A partir del análisis de los diferentes planes y proyectos fue posible establecer relaciones entre ellos (influencias de unos sobre otros, lógicas proyectuales comunes, incidencia de los cambios organizativos...). Son estas interacciones las que configuran las visiones generales sobre los procesos de renovación del espacio público. Ahora bien, cabe preguntarse si estos procesos han constituido auténticas políticas de espacio público, actuaciones coordinadas y coherentes en el tiempo. O si han dado lugar a culturas del espacio público, a saberes compartidos que se van acumulando y enriqueciendo para ejercer una influencia sobre el rumbo de las intervenciones futuras. ¿Cuáles han sido las lógicas dominantes en estas culturas del espacio público: el tratamiento de los espacios cómo objetos singulares de diseño; unas lógicas urbanas que atienden al papel de los espacios en relación con el conjunto de la ciudad; lógicas más funcionales que se fijan sobretodo en los requerimientos de los usuarios? Son algunas de las preguntas que responde la investigación y que permiten comprender para cada ciudad la experiencia de renovación de su espacio público y compararla con la del resto de ciudades. Cada ciudad presenta su propia experiencia, pero a la vez se ponen de manifiesto influencias de algunas sobre otras, y rasgos comunes entre todas ellas, resultado de haber interactuado en contextos urbanos similares (de una gran riqueza patrimonial y con administraciones públicas capaces de ejercer el liderazgo de las transformaciones urbanas).

Paralelamente, desde la línea de investigación se impulsaron nuevas tesis doctorales sobre nuevas tipologías de espacios de encuentro en la ciudad contemporánea, más recientes en su aparición y extensión que los espacios urbanos más tradicionales que dominaban el estudio en las ciudades compactas. Se trataba de extender el conocimiento de los espacios sociales al conjunto del jardín de la metrópolis. Entre estas tipologías se identificaron tres: los espacios colectivos, los espacios infraestructurales y los grandes espacios libres metropolitanos.

El estudio de los espacios colectivos ya había sido considerado como una prioridad por Manuel de Solà-Morales en 1992 cuando publicaun artículo en La Vanguardia titulado precisamente "Espacios colectivos: urbanizar lo privado". Dentro de la línea de investigación de espacio público se está realizando una 
tesis doctoral a cargo de Pedro Bento bajo la dirección de Estanislau Roca sobre dicho tema. Esta propuesta de tesis tiene como objeto de estudio los grandes equipamientos urbanos de ocio y consumo -centros comerciales o shopping centers y otras tipologías más recientes como los centros de ocio o de entretenimiento- que se implantan dentro de la ciudad compacta. Los objetivos son incrementar el conocimiento sobre el tema de los espacios colectivos y los centros comerciales en particular, y evaluar las oportunidades y amenazas que los mismos puedan o no representar. Pensamos que en determinadas circunstancias estos artefactos no solamente crean nuevos lugares en la ciudad compacta, sino que califican y enriquecen el sistema de los espacios públicos donde se insertan, funcionando, incluso, como catalizadores o motores del desarrollo y transformación urbanística. La tesis propone un análisis exhaustivo de los tipos de espacios colectivos generados en el interior de los artefactos comerciales y de ocio, de los grados de libertad, de las formas de apropiación, de las permeabilidades visuales, de los accesos/espacios de transición/cruces, de su impacto sobre la dinámica edificatoria, el espacio público y la actividad económica en su entorno. La tesis procurará demostrar que estos objetos son equipamientos importantísimos para nuestras ciudades. Aunque sean objeto del perjuicio social por un tema que nada tiene que ver con la arquitectura o el urbanismo, sino única y exclusivamente con la propiedad privada de la inmensa mayoría de ellos, estos objetos responden a las necesidades crecientes de ocio y consumo de las sociedades contemporáneas.

El estudio de los corredores infraestructurales en el territorio también había sido objeto de una reflexión por parte de Manuel de Solà-Morales en otro artículo aparecido en La Vanguardia: "Un nuevo paseo de Gracia". La tesis de Joan Moreno, dirigida por Estanislau Roca, se interesa por los nodos de ordenación del territorio metropolitano, a los que llama esquinas metropolitanas, comparando las regiones del Randstad y Barcelona.

Las redes de transporte de personas, bienes y energía son los elementos estructurantes de la metrópolis contemporánea. El territorio de las redes se ordena a partir de canales de gestión de los flujos y de nodos de intercambio. El diseño de los canales de transporte, de acuerdo con criterios de eficiencia, y la distribución de los nodos de actividad, que priorizan la conectividad a una red multiescalar, generan fricción con el territorio soporte. La segregación funcional de actividades y movilidad, defendidas por el Movimiento Moderno, ha sido el fundamento de las políticas urbanísticas de la segunda mitad del siglo XX. En la actualidad se prioriza la compatibilidad entre el derecho a la movilidad y el derecho al territorio. Acordar el desarrollo de la estructura social y económica con el reforzamiento de las cualidades propias del medio es el reto presente en la revisión de los modelos de ordenación territorial. La coordinación entre los sistemas de transporte en los accesos a la red y las actividades antrópicas, deben permitir mejorar tanto la optimización de las redes, como la ocupación del territorio. Los nexos de conexión con las estaciones ferroviarias y los nudos viarios se convierten en espacios de 
centralidad de acuerdo con criterios cualitativos de ordenación: son nuevas esquinas metropolitanas.

El Randstad holandés es una metrópoli policéntrica con una jerarquía urbana excepcional en el occidente europeo. Las redes han ido modelando, desde su origen, un territorio condicionado por una ficticia tabula rasa. Programas de ordenación urbana en el entorno de los nodos de transporte han sido el ámbito de experimentación de referencia para otros territorios en proceso de dispersión como Barcelona-Región. La administración de los territorios metropolitanos a partir de regiones-corredor especializadas debe contribuir a la optimización de la red de transporte, la preservación de los suelos heredados con valores cualitativos y el desarrollo de actividad de modo coordinado entre nodos territoriales.

Simultáneamente al estudio de las infraestructuras en las regiones metropolitanas se está impulsando también una investigación sobre el impacto de las infraestructuras dentro de la ciudad compacta. La tesis de Daniel Mòdol, dirigida por Estanislau Roca, investiga precisamente las relaciones entre la infraestructura y el espacio público en el contexto urbano, entendiendo estas arquitecturas de los sistemas como la base del desarrollo del plano de relación de la ciudad y un elemento capaz de fijar y determinar los escenarios urbanos donde están presentes. La situación de proximidad, pero también de integración entre las soluciones a la movilidad y la calificación del espacio público, ha generado escenarios mutantes que ilustran buena parte de la historia del urbanismo en nuestras ciudades. Del análisis de la evolución formal y funcional de estos paisajes ligados a la movilidad, en sus diferentes focalizaciones tanto infraestructurales, como de especialización del plano de la ciudad, la tesis espera extraer criterios de diseño exitosos para la correcta proyectación futura, tanto de escenarios ex-novo, como de la reforma de espacios urbanos existentes. Comparando la evolución que distintos espacios de una misma ciudad (Barcelona, en este caso) han experimentado a lo largo de los últimos 150 años, descubrimos el papel fundamental que han jugado en todos ellos la resolución de la movilidad, la integración de las infraestructuras circulatorias y los criterios de proyecto del espacio público en constante revisión. Del análisis conjunto de todos estos casos de estudio, se puede concluir que probablemente nos estamos acercando a una nueva concepción de la estructura urbana de la ciudad, donde (al lado de los elementos más habituales de calificación de los espacios del plano de la ciudad) se incorporan lógicas y funcionalidades que, procedentes del subsuelo, adquieren un papel absolutamente fundamental en la proyectación de la ciudad moderna del futuro.

Al lado de los espacios colectivos y los espacios infraestructurales, un tercer gran tipo de espacios públicos contemporáneos serían los grandes espacios libres metropolitanos, áreas naturales antropizadas rodeadas por los crecimientos metropolitanos que amenazan con fagocitarlos progresivamente, pero que a un mismo tiempo incrementan y diversifican sus usos, 
convirtiéndolos en auténticos espacios públicos. En la región metropolitana de Barcelona, el curso bajo del río Llobregat y su propio delta serían ejemplos de estos espacios, en los que los ecosistemas naturales, las actividades agrícolas y el ocio urbano conviven e interactúan. El estudio de estas áreas, base de la matriz del jardín de la metrópolis, ha formado también parte de la línea de investigación. Por el momento, existe una propuesta de tesis incipiente (a cargo de Marina Matashova), que estudia los espacios libres dentro de las zonas ribereñas del Llobregat entre Martorell y Sant Boi. Las relaciones entre los espacios fluviales y los espacios públicos urbanos de las localidades situadas en el valle, así como la identificación de espacios con potencial para acoger nodos de actividad que favorezcan la integración de las franjas fluviales al conjunto de la conurbación metropolitana, son algunos de los aspectos abordados en la investigación.

A lo largo del desarrollo de estos trabajos, especialmente los relacionados con los espacios colectivos y con los espacio infraestructurales, se ha puesto en evidencia la importancia en la ciudad contemporánea de los espacios híbridos. En estos casos, espacios libres y espacio construido se articulan e imbrican en diferentes niveles y planos, generando la arquitectura de un proyecto urbano tridimensionalmente complejo. Estos espacios híbridos han sido objeto de interés dentro de la línea de investigación, lo que ha dado lugar a otra tesis incipiente que estudia los espacios públicos en Hong Kong, a cargo de Francesco Rossini y dirigida por Estanislau Roca. Los espacios públicos interiores situados a diferentes niveles dentro de los edificios o los sistemas de pasarelas peatonales elevadas que los comunican son ejemplos de estos espacios híbridos que ya marcan el paisaje de algunas ciudades asiáticas.

Un último aspecto que se ha trabajado dentro de la línea de investigación sobre espacio público son sus significados, y especialmente la posibilidad que el espacio público siga generando sentidos y experiencias cívicas. La tesis doctoral de Miquel Martí, "En búsqueda de la civitas contemporánea", hacia especial hincapié en este aspecto. En ella se aborda cómo desde un política municipal de espacio público que ponga énfasis en la lógica urbana se pueden lograr espacios públicos plenamente contemporáneos que sigan siendo escenarios para la ciudad y la vida que en ella se desarrolla, y que funcionen como metáforas inconscientes del marco social que hace posible la convivencia en un contexto de máxima diversidad. A través de la tesis doctoral en curso de Ana Paula Montes, codirigida por Miquel Martí y Zaida Muxí, se están explorando aquellas otras formas de generar sentido cívico que no proceden de las políticas lideradas por la administración, sino por las diferentes maneras de autogestión del espacio (desde las ocupaciones alternativas de espacios no proyectados de la ciudad hasta los mecanismos de autoconstrucción del espacio en economías informales). La preocupación por la significación cívica del espacio público ha sido un aspecto subyacente a toda la línea de investigación. 


\section{PERSPECTIVAS DE FUTURO PARA LA LÍNEA DE INVESTIGACIÓN SOBRE EL ESPACIO PÚBLICO Y SU PROYECTO URBANO}

El espacio público, los lugares contemporáneos que propician el encuentro y la interacción social entre ciudadanos, es desde hace unas décadas un tema central de la investigación en urbanismo. Desde la línea de investigación sobre el espacio público del Departamento de Urbanismo y Ordenación del Territorio de la UPC se quiere potenciar este ámbito de estudio a partir de cuatros grandes premisas.

En primer lugar, es importante seguir teniendo en cuenta la diversidad de espacios públicos contemporáneos. Las investigaciones deben abarcar los espacios característicos tanto de la ciudad compacta (espacios públicos, colectivos e infraestructurales urbanos), como los de la ciudad extendida por el territorio (corredores y nodos infraestructurales, grandes espacios libres metropolitanos, espacios residuales de la baja densidad...).

En segundo lugar, para cada tipo de espacio estudiado es importante tener una visión de conjunto de los procesos de su transformación. No sólo interesarse por el análisis de los resultados proyectuales, sino también por las lógicas que los animan y los agentes que los protagonizan.

En tercer lugar, esta visión de conjunto implica estar también atentos a las vivencias que se producen en los espacios públicos estudiados. Desde sus comienzos, la línea de investigación ha estado abierta a los significados que el espacio público es capaz de generar. Sin olvidar que parte de una aproximación claramente morfológica, tampoco puede menoscabar el hecho que el estudio del espacio público es substancialmente multidisciplinar Existen aportaciones esenciales desde muchos campos disciplinares (filosofía, sociología, antropología, historia...), que el urbanista debe conocer para entender el espacio público contemporáneo. Es así cómo los procesos de apropiación del espacio público, las vivencias cotidianas, la festivalización mediante acontecimientos singulares, entre otros, son aspectos que entran de lleno en el centro de interés de la línea de investigación.

Esta voluntad de aportar una visión de conjunto sobre el fenómeno del espacio público queda reflejada en la asignatura del Máster Universitario en Urbanismo, la cual, bajo el título "Espacios públicos: vivencias, proyectos y gestión", quiere ser una plataforma que enriquezca y al mismo tiempo visualice los resultados de la línea de investigación. En esta asignatura se trabaja con referencias bibliográficas multidisciplinares que permiten abordar las numerosas experiencias semánticas a las que da lugar el espacio público, la diversidad de tipologías y los diferentes mecanismos proyectuales para cada una de ellas dentro del complejo mundo del proyecto urbano del espacio público, y atender a la dimensión organizativa y de gestión de los procesos de renovación, la cual, ya sea en contextos altamente institucionalizados o en contextos más informales, tiene una gran incidencia en la forma final del espacio público. 
Por último, la línea de investigación apuesta por realizar estudios en contextos culturales diversos. Hasta el momento el ámbito europeo ha sido el más trabajado, con la investigación sobre políticas de espacio público en ciudades europeas llevada a cabo por Miquel Martí, o las tesis de Joan Moreno y Pedro Bento que comparan casos catalanes con otros holandeses y portugueses respectivamente. Es fundamental ampliar los estudios a contextos que presentan mayores diferencias en relación con el espacio público, como pueden ser la ciudad norteamericana, la ciudad informal en Latinoamérica o África, o la ciudad asiática. Las estancias realizadas por Pep Fortià y Miquel Martí en EE.UU. durante la elaboración de sus tesis doctorales, la estancia de investigación llevada a cabo por el propio Miquel Martí en Shanghai y Kyoto en 2010, o las tesis doctorales en curso de Francesco Rossini sobre los espacios híbridos en Hong Kong y de Arwen Prendersgast sobre la producción del espacio público en ciudades altamente informales como Maputo en Mozambique, son unos primeros intentos de internacionalización de la línea de investigación. Nuevas tesis doctorales sobre casos de estudio en diferentes continentes, trabajos post-doctorales que comparen contextos culturales diversos o proyectos de investigación internacionales son algunas de las vías que se plantean para que la línea de investigación sobre espacio público del DUOT pueda llegar combinar una visión a la vez profunda sobre los casos de estudio, general sobre las dimensiones que intervienen en la transformación del espacio público y global sobre distintos contextos urbanísticos y culturales.

Estanislau Roca Blanch, Arquitecto Doctor en Urbanismo Profesor del programa de Doctorado en Urbanismo Departament d'Urbanisme i Ordenació del Territori. Universitat Politècnica de Catalunya jane2@coac.net 


\section{Referencias bibliográficas}

\section{Sentidos cívicos del espacio público contemporáneo}

BERMAN, Marshall (1991). Todo lo sólido se desvanece en el aire : la experiencia de la modernidad. Madrid: Siglo Veintiuno. HABERMAS, Jürgen (1981). Historia y crítica de la opinión pública: la transformación estructural de la vida pública. México D.F.: Gustavo Gili.

HAJER, Maarten y REIJNDORP, Arnold (2001). In search of a new public domain : analysis and strategy. Rotterdam : NAi.

SENNETT, Richard (1997). Carne y piedra: el cuerpo y la ciudad en la civilización occidental. Madrid: Alianza,.

\section{¿La muerte del espacio público?}

AUGÉ, Marc (1993). Los "no lugares". Espacios del anonimato: una antropología de la sobremodernidad. Barcelona: Gedisa.

CASTELLS, Manuel (1998). "Espacios públicos en la sociedad informacional" en Subirós, Pep (ed.), Ciutat real, ciutat ideal: significat i funció a l'espai urbà modern. Barcelona: CCCB.

GHORRA-GOBIN, Cynthia (ed.)( 2001). Réinventer le sens de la ville : les espaces publics à l'heure globale. Paris : L'Harmattan.

KOOLHAAS, Rem (2006). La ciudad genérica. Barcelona, Gustavo Gili.

SORKIN, Michael (ed.) (2004). Variaciones sobre un parque temático: la nueva ciudad americana y el final del espacio público. Barcelona: Gustavo Gili.

\section{Uso cotidiano del espacio público}

DE CERTEAU, Michel (1996-99). La invención de lo cotidiano. Mexico D.F.: Universidad Iberoamericana.

DELGADO, Manuel (1999). El animal público: hacia una antropología de los espacios urbanos. Barcelona: anagrama.

DELGADO, Manuel (2007). Sociedades movedizas: pasos hacia una antropología de las calles. Barcelona: anagrama.

SENNETT, Richard (1978). El declive del hombre público. Barcelona: Edicions 62.

SENNETT, Richard (1991). La conciencia del ojo. Barcelona: Versal.

\section{Apropiación del espacio público}

BORJA, Jordi (2003). El espacio público: ciudad i ciudadanía. Barcelona: Electa.

CHASE, CRAWFORD, KALISKI (1999). Everyday urbanism. New York: Monacelli.

GEHL, Jan (2006). La humanización del espacio urbano: la vida social entre los edificios. Barcelona: Reverté.

LEFEBVRE, Henri (1969). El derecho a la ciudad. Barcelona: Península.

SABATÉ, Joaquim (2004). Event places. Barcelona: UPC.

\section{Lógicas de proyectación del espacio público}

AA.DD. (2010). In favour of public space. Barcelona: CCCB.

CHOAY, Françoise (2007). Alegoría del patrimonio. Barcelona: Gustavo Gili.

GEHL, Jan (2002). Nuevos espacios urbanos. Barcelona: Gustavo Gili.

MARTÍ, Miquel. A la recerca de la civitas contemporània. Cap a una cultura urbana de l'espai públic: l'experiència de Barcelona. DUOT (Tesis Doctoral).

MARTíN, Ángel (2004). Lo urbano en 20 autores contemporáneos. Barcelona: Edicions UPC.

ROCA, Estanislau et alt. Campus de la Diagonal. Un projecte urbà : praxi docent i professional. Barcelona : Edicions UB, 2009.

ROCA, Estanislau et alt (2010). Diagonal, un procés urbà : el projecte urbà en la reforma de la ciutat. Barcelona: Edicions ETSAB.

ROCA, Estanislau et alt (2011). Glòries, reforma urbana i espai públic. Barcelona: Edicions ETSAB.

SOLÀ-MORALES, Manuel (2008). De cosas urbanas. Barcelona: Gustavo Gili.

www.pps.org 